\title{
Wild edible plants as potential antioxidant or nutritional supplements for beverages minimally processed
}

\author{
Paloma Sanchez-Bel a, ${ }^{\text {, }}$, Ana Romojaro b, ${ }^{\text {, Isabel Egea a }}$, Maria Teresa Pretel ${ }^{\text {b, * }}$ \\ a Departamento Biología del Estrés y Patología Vegetal, Centro de Edafología y Biología Aplicada del Segura, CSIC, Espinardo, Murcia, Spain \\ ${ }^{\mathrm{b}}$ Fisiología Vegetal, Botánica. Dpto. Biología Aplicada, Escuela Politécnica Superior de Orihuela, Universidad Miguel Hernández, Ctra Beniel-Orihuela, \\ Km 3.2, 03312 Orihuela, Alicante, Spain
}

\section{A R T I C L E I N F O}

\section{Article history:}

Received 28 January 2013

Received in revised form 3 June 2014

Accepted 4 June 2014

Available online xxx

\section{Keywords:}

Wild edible plants

Nutritional supplement

Antioxidant potential

Fruit

Minimal processing

\begin{abstract}
A B S T R A C T
Wild edible plants (WEP) with high antioxidant capacity, Rosa canina fruits, Quercus ballota acorns and Sanguisorba minor (young leaves and stems), were added to orange and kiwifruit juices for increase the nutritional properties. The addition of WEP to orange juice (OJ) and kiwifruit cremogenate (KC) does not affect, or it can even improve, some parameters of consumer's acceptance, although the mixtures increasing sweetness and intensifying the matrix colour without changing the tone are the best valued. The beverages minimally processed with higher TSS were the matrices fortified with $Q$. ballota, the same that were considered sweetest by the judges. The addition of WEP to OJ did not significantly change the levels of vitamin C (ascorbic acid plus dehydroascorbic acid). The addition of $R$. canina and $S$. minor increased the content of phenolic compounds and antioxidant capacity in both matrices (OJ and $\mathrm{KC}$ ) in more than $30 \%$. Beverages with a high interest for consumers and with high antioxidant properties have been obtained.
\end{abstract}

() 2014 Elsevier Ltd. All rights reserved.

\section{Introduction}

During the last years, both food quality and its effects on human health has become a fundamental issue. As a result, many different research works about the functional properties of different foods, plant extracts and natural ingredients have been developed (Serrano et al., 2009). This new awareness have taken food industry to focus the attention on the development of functional or nutraceutical foods with beneficial properties for health (Lavecchia, Rea, Antonacci, \& Giardi, 2013). Some years ago, the compounds with nutraceutical activity added to foods were usually chemically produced, but new demands for a sustainable life have made that the food industry is now using natural compounds, mainly those derived from plants. Therefore, we can now find in the market a great variety of functional products consisting of food matrices to which extracts of botanical species with known functional and nutraceutical properties have been added (González-Molina, Moreno, \& García-Viguera, 2008).

In this regard, there is a great variety of wild non-cultivated plants that have been employed in the diet and the traditional

\footnotetext{
* Corresponding author. Fax: +34 966749678.

E-mail address: mteresa.pretel@umh.es (M.T. Pretel).

1 Both authors contributed equally to this work.
}

medicine of the Mediterranean region (Pardo de Santayana, Tardío, \& Morales, 2005), but their use has now decreased, an even disappeared, as a consequence of the industrial revolution. Among them, Rosa canina L., Quercus ballota Desf. and Sanguisorba minor Scop., are good candidates for their study as possible functional ingredients due to the nutraceutical and therapeutic properties that many recent studies have found in them. $R$. canina is a significant source of tocopherols, flavonoids, carotenoids and ascorbic acid (Barros, Carvalho, \& Ferreira, 2011) and this composition is probably the reason for its high antioxidant capacity (Egea, Sánchez-Bel, Romojaro, \& Pretel, 2010). $R$. canina also presents antiinflammatory (Lattanzio et al., 2011) and antibacterial activities (Yilmaz \& Ercisli, 2011), anticholinesterase capacity (Boğa, Hacıbekiroğlu, \& Kolak, 2011) and antidiarrhoeal properties (Mandade, Choudhury, Harsulkar, \& Wakade, 2011). On the other side, the fruits of $Q$. ballota are rich in $\alpha$ - and $\gamma$-tocopherol and phenolic compounds, especially hydrolysable tannins (Tejerina, García-Torres, Cabeza de Vaca, Vázquez, \& Cava, 2011). Q. ballota also presents a high antioxidant capacity (Romojaro, Botella, Obón, \& Pretel, 2013; Tejerina et al., 2011), and the antioxidant activity of the acorn powder has been even characterised in order to be used as a new natural antioxidant for fats and oils (Lee, Jeong, \& Oh, 1992). Regarding S. minor, it has been already shown that its young leaves and stems have many and varied phenolic compounds 
that are derivatives of caffeic acid, apigenin, quercetins, kaempferol with a high antioxidant and antimycotic activities (Gatto et al., 2011; Romojaro et al., 2013).

Fruit juices are usually employed as food matrices for the production of functional foods. In fact, recent studies have shown that the beneficial properties of fruit juices can be increased by adding antioxidant sources (González-Molina et al., 2008). Orange (Citrus cinensis) is a common raw material for the world fruit juice industry and its juice has a high nutritional value due to bioactive compounds, as well as an appreciated flavour and aroma (Pala \& Toklucu, 2013). The kiwifruit has also a great potential for industrial exploitation, mostly due to its composition rich in phytonutrients (Tavarini, Degl'Innocentia, Remorinib, Massaib, \& Guidi, 2008). However, the obtaining of a good quality processed kiwifruit is not simple and the possibility to have an industrial valorisation of the fruit is yet away to be found. The main goal of the kiwifruit processing is to obtain safe and stable products able to retain as much as possible the peculiarity of fresh fruit, including green colour, aroma, nutritional value and structural characteristics. One of the main limitations for obtaining kiwifruit minimally processed is the difficulty for maintaining the original green colour which, due to oxidation, quickly acquires brownish tonalities (Benlloch-Tinoco, Igual, Rodrigo, \& Martínez-Navarrete, 2013; Cassano \& Drioli, 2007).

Food industry is currently focused on the development of novel and minimally processed products with improved quality. This type of products has been traditionally preserved by means of conventional thermal technologies. However, it usually requires the use of high temperatures combined with long process times which has been widely associated with a marked deterioration in food quality, especially with the development of cooked off-flavours, colour alteration and the loss of thermosensitive compounds (BenllochTinoco et al., 2013). For this reason, alternatives to conventional processing technologies are being explored. The addition of extracts of wild plants with high antioxidant capacity could, apart from increasing the nutritional value of the juices, help to stabilize and even improve their valued organoleptic properties.

Considering all the previous background, the main aim is to study if the incorporation of R. canina, Q. ballota and S. minor to orange and kiwifruit juices minimally processed could increase the nutritional properties and stabilize or even improve their organoleptic characteristics.

\section{Materials and methods}

\subsection{Wild edible plants (WEP)}

The non-cultivated and traditionally collected wild edible plants were harvested in the South East (SE) of Spain. Plants were frozen in liquid nitrogen, lyophilized and powdered using a domestic mixer and, finally, stored at $20^{\circ} \mathrm{C}$ until further analysis. Wild edible plants used in this study were $R$. canina (fruits), Quercus ballota (fruits) and Sanguisorba minor (young leaves and stems).

\subsection{Fruit matrices}

For the making of kiwifruit cremogenate (KC), kiwifruit of variety Hayward (Actinidia deliciosa) from Galicia (España) was employed. For the making of orange juice (OJ), oranges of variety Navel Lane-Late (Citrus sinensis) from Valencia (España) were employed. Both kiwi and orange fruits were purchased at commercial maturity state. The orange juice was obtained using a domestic squeezer (Princesa, Barcelona, Spain) and the kiwifruit cremogenate was obtained using a domestic blender (Osterizer,
Madrid, Spain). Both fruits (kiwi and orange) were processed to make juice at eating ripeness.

\subsection{Preparation of enriched beverages minimally processed}

The lyophilised WEP were added to the fruit matrices: natural orange juice $(\mathrm{OJ})$ and kiwifruit cremogenate $(\mathrm{KC})$ and they were homogenised in polytron (Kinematica AG, Lucerne, Switzerland). Previous experiments performed in our laboratory adding $0.1,0.5,1$ and $2 \mathrm{~g}$ of WEP per $100 \mathrm{~mL}$ of matrix, showed that the best organoleptic results were obtained when proportion of $0.5 \mathrm{~g}$ $100 \mathrm{~mL}^{-1}$ was used. Thus this was the proportion used in the subsequent experiments. Homogenised mixtures were filtered in a gauzed filter and stored at room temperature for $24 \mathrm{~h}$. Samples were analysed after filtering (time 0 ) and after $24 \mathrm{~h}$ of storage.

\subsection{Sensory determination}

Sensory evaluation was conducted by a selected and trained panel comprising ten judges (seven women and three men between twenty and fifty years old) in accordance with ISO 8586$1: 1993$. The tasting room was kept at $20^{\circ} \mathrm{C}$ and beverages served in tasting glasses certified and coded. The evaluation was done using 10 -point structured scales, 10 being the best and 1 the worst quality (colour, acidity, sweetness and aroma). The general acceptance quality attribute was assessed as the measurement of the acceptability of the product by the consumer using a scale from "very unpleasant" (level 1) to "very pleasant" (level 10). The selection and training of tasters, as well as the fitting-out of the tasting room, were carried out according to Sanchez-Bel, Egea, Romojaro, and Martinez-Madrid (2008). The purchase intent was also evaluated. Results were expressed as percentage of acceptance being 100 the punctuation obtained by the unfortified juice matrix.

\subsection{Quality parameters (TSS, $p H, T A)$}

The total soluble solids content (TSS) was determined by measuring the refractive index of the juice from the fruit with a digital refractometer (Atago, Tokyo, Japan). TSS content was expressed as percentage. $\mathrm{pH}$ was measured in a pHmeter (Crison, Barcelona, Spain). Titratable acidity (TA) was determined by


results were expressed as grams of malic acid per $100 \mathrm{~g}$ of fresh weight.

\subsection{Colour quality}

Colour was measured with a CR-400 colorimeter (Minolta, Paris, France) and results were expressed in CIELAB units of $L^{*}, a^{*}$ and $b^{*}$. Hue angle $(H)$ and chroma $(C)$ were computed from $L^{*}, a^{*}$ and $b^{*}$ as $H=\operatorname{tg}^{-1}\left(b^{*} / a^{*}\right)$ and $C=\sqrt{ }\left(a^{*}\right)^{2}+\left(b^{*}\right)^{2}$.

\subsection{Determination of vitamin C (ascorbic and dehydroascorbic acid)}

Fruit ascorbate (ASC) content was determined according to Egea et al. (2010) with some modifications; $3 \mathrm{~mL}$ of samples homogenised in $10 \mathrm{~mL}$ of $5 \mathrm{~g} 100 \mathrm{~mL}^{-1}$ cold metaphosphoric acid and stirred continuously for $30 \mathrm{~min}$ at $4{ }^{\circ} \mathrm{C}$ in darkness, and then centrifuged at $20,000 \mathrm{~g}$ for $25 \mathrm{~min}$. The supernatant was passed through a C18 column (Sep-pack plus, Waters, Barcelona, Spain) and a $0.2 \mu \mathrm{m}$ filter. The filtered supernatant was used for ascorbic acid determination using HPLC (LC-10Atvp, Shimadzu, Barcelona, Spain) with a thermostated ion-exchange column (ION-300) at $30{ }^{\circ} \mathrm{C}$ and isocratic elution. The absorbance was recorded with a 
UV/vis detector at $245 \mathrm{~nm}$. A standard curve in the range $10-100 \mathrm{mg} \mathrm{kg}^{-1}$ ascorbic acid was used. The results were expressed as grams of ascorbic acid per $100 \mathrm{~g}$ of fresh weight.

Total ascorbate content was determined after reduction of dehydroascorbate (DHA) to ASC with DTT in a final concentration of $30 \mathrm{mmol} \mathrm{L}^{-1}$ for $8 \mathrm{~h}$ in the dark, and then measured as ASC following re-chromatography. Concentration of DHA was estimated from the difference between total ascorbate pool (ASC plus DHA) and ASC.

\subsection{Determination of total phenolic compounds}

Total phenolic compounds were determined according to Serrano et al. (2009) using (3 g) homogenized in $10 \mathrm{~mL}$ of $100 \mathrm{mmol} \mathrm{L}^{-1}$ sodium phosphate buffer containing $150 \mathrm{mmol} \mathrm{L}^{-1}$ $\mathrm{NaCl}, \mathrm{pH} 7.4$ on ice. The concentration of the total phenolic compounds in fruit extracts was determined by comparison with the absorbance of analytical grade phenol standard, gallic acid, at different concentrations. The results were expressed as grams of galic acid per $100 \mathrm{~g}$ of fresh weight (FW).

\subsection{Determination of antiradical activity: Trolox equivalent antioxidant capacity (TEAC assay)}

The $\mathrm{ABTS}^{\bullet-}$ radical anion solution was generated by incubating, at $60{ }^{\circ} \mathrm{C}$ for $6 \mathrm{~min}$, a mixture of $2.5 \mathrm{mmol} \mathrm{L}^{-1} 2,2^{\prime}$-azobis(2amidinopropane) hydrochloride (ABAP) and $20 \mathrm{mmol} \mathrm{L}^{-1} 2,2^{\prime}$ azinobis(3-ethylbenzothiazoline-6-sulfonate) $\left(\mathrm{ABTS}^{2-}\right.$ ) stock solution in $100 \mathrm{~mL}$ of phosphate buffer $\left(100 \mathrm{mmol} \mathrm{L}^{-1}\right.$ phosphate and $150 \mathrm{mmol} \mathrm{L}^{-1} \mathrm{NaCl}$, pH 7.4). Samples (3 g) were homogenized in $10 \mathrm{~mL}$ of $100 \mathrm{mmol} \mathrm{L}^{-1}$ sodium phosphate buffer containing $150 \mathrm{mmol} \mathrm{L}^{-1} \mathrm{NaCl}$, pH 7.4 on ice. The supernatant was collected as final extract. $40 \mu \mathrm{L}$ of each extract were mixed with $1960 \mu \mathrm{L}$ of the radical solution to measure the antioxidant activity at $734 \mathrm{~nm}$ after 6 min of incubation at $36^{\circ} \mathrm{C}$. A calibration curve was prepared with different concentrations of Trolox. The TEAC activity was calculated according to Egea et al. (2010) and represents the concentration of Trolox, in $\mu \mathrm{mol} \mathrm{L} \mathrm{L}^{-1}$, that has the same antioxidant capacity as the analysed sample (Trolox equivalent).

\subsection{Statistical analysis}

Tests for significant differences were carried out using the general linear model of the SPSS (version 14.0, SPSS Inc., Chicago, USA) statistical package. Analysis of variance (ANOVA) was conducted using the treatments as a statistical parameter, the confidence level being 95\%. Correlations between different parameters were computed as Pearson's correlation coefficient $(r)$. When differences were significant, multiple comparisons test was made using least significant difference test (LSD). Values represent the means \pm SD of three replications for each of three sub-samples per sample.

\section{Results and discussion}

\subsection{Consumers' acceptance}

The sensory assessment is the main aspect for designing new foods because changes in aroma, texture or colour of the original matrices can significantly affect the consumer's acceptance. Although the overall impression is a function of many attributes and their interactions, it is interesting to identify the particular attributes that move acceptance. The complexity of food is such that it may well be impossible to truly discern the relative contributions of the different sensory inputs to food acceptance (Janzantti, Machado, \& Monteiro, 2011). In this work, the attribute colour was assessed in different ways according to the matrix (OJ or $\mathrm{KC})$ and the added WEP ( $R$. canina -Rc-, Quercus ballota $-\mathrm{Qb}$ - or Sanguisorba minor -Sm-) (Table 1). The colour of the OJ fortified with $R c$ was significantly $(p<0.05)$ better valued than the control one (119.72 \pm 5.31$)$, while the addition of $Q b$ did not present differences regarding control OJ $(95.87 \pm 3.73)$. The addition of $\mathrm{Sm}$ to OJ reduced the redness of the orange juice and this fact was negatively assessed by the consumer $(39.14 \pm 10.59)$. Regarding KC, consumers positively assessed the increase of green colour intensity provided by $S m(124.01 \pm 8.73)$. On the contrary, the addition of $R c$ gave KC a brownish aspect that was significantly worse valued $(61.86 \pm 9.42)$ than that of control KC. All matrices were considered less acid than control, regardless of the added WEP. The WEP addition increased the sweetness between 35 and $85 \%$ regarding the unfortified matrices. The addition of WEP to OJ and KC decreased the aroma assessment in most mixtures, although not significantly. In our work, and in keeping with other authors (Tepper, 1993) regarding overall impression, colour was the most significant value because the worst assessed mixtures were OJ with $\mathrm{Sm}$ and KC with $R c$, a fact coincident to the worst assessment of colour. The best valued were OJ with $R c$ and KC with Sm. Consumers showed a better purchase intent for OJ with Rc and KC with Sm and Qb.

It is worth mentioning the high purchase intent of $K C$ with $Q b$ because it would be expected that this enriched beverage show an increase of an astringent and bitter flavour due to the high content of tannins. However, matrices with $Q b$ obtained a better overall impression and purchase intent than KC. Some authors have stated that the astringent flavour can be modified by the addition of other compounds, and that the sweetness can reduce the astringent sensation. In this study, matrices with $Q b$ were those obtaining the highest punctuation in sweetness, a fact stating that, despite the high content in tannins of $Q b$, the extra supply of sweetness can avoid the astringent and bitter flavour of the tannins. In general, it

Table 1

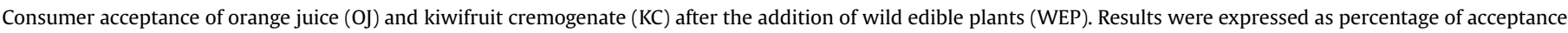
being 100 the punctuation obtained by the unfortified juice matrix.

\begin{tabular}{|c|c|c|c|c|c|c|}
\hline Matrix with WEP ${ }^{\mathrm{a}}$ & Colour $^{\mathrm{b}}$ & Acidity $^{\mathrm{b}}$ & Sweetness $^{\mathrm{b}}$ & Aroma $^{\mathrm{b}}$ & Overal impression $^{\mathrm{b}}$ & Purchase intent $^{\mathrm{b}}$ \\
\hline OJ with $R c$ & $119.72 \pm 5.31$ & $87.36 \pm 5.81$ & $153,57 \pm 17.45$ & $84.76 \pm 12.42$ & $103.45 \pm 8.35$ & $117.62 \pm 9.79$ \\
\hline OJ with $Q b$ & $95.87 \pm 3.73$ & $92.08 \pm 7.04$ & $184.72 \pm 31.93$ & $83.57 \pm 6.08$ & $100.87 \pm 9.40$ & $95.24 \pm 5.53$ \\
\hline OJ with Sm & $39.14 \pm 10.59$ & $85.92 \pm 7.95$ & $155.56 \pm 35.44$ & $80.95 \pm 11.28$ & $80.00 \pm 24.34$ & $89.76 \pm 18.6$ \\
\hline KC with $R c$ & $61.87 \pm 9.42$ & $63.45 \pm 10.67$ & $135.75 \pm 14.75$ & $95.04 \pm 5.72$ & $84.01 \pm 8.70$ & $65.43 \pm 15$ \\
\hline KC with $Q b$ & $114.34 \pm 6.97$ & $84.64 \pm 10.76$ & $157.01 \pm 30.06$ & $82.54 \pm 10.78$ & $125.1 \pm 15.5$ & $115.00 \pm 14$ \\
\hline KC with Sm & $124.01 \pm 8.73$ & $75.00 \pm 6.96$ & $152.38 \pm 35.62$ & $105.79 \pm 23.85$ & $129.6 \pm 11.8$ & $123.00 \pm 17$ \\
\hline$L S D(p<0.05)^{c}$ & 11.67 & 10.67 & 40.44 & 17.31 & 29.09 & 15.17 \\
\hline
\end{tabular}

${ }^{a}$ Matrix: Orange juice (OJ), kiwifruit cremogenate (KC) fortified with WEP (Rosa canina -Rc-, Quercus ballota -Qb- or Sanguisorba minor -Sm-).

b Colour, acidity, sweetness, aroma, overall impression and purchase intent. (100 the punctuation obtained by the unfortified matrix).

c $L S D(p<0.05)$, least significant difference, was used for significant difference comparison among means of various samples. 
could be concluded that the addition of WEP to OJ and KC does not affect, or it can even improve, some parameters of consumer's acceptance, although the mixtures increasing sweetness and intensifying the matrix colour without changing the tone are the best valued.

\subsection{Changes in quality parameters}

The addition of WEP to matrices (OJ and $\mathrm{KC}$ ) did not significantly change $(p<0.05)$ the $\mathrm{pH}$ or the TA of the mixtures (Table 2$)$. The TA of the new beverages ranged between $1.66 \pm 0.04$ of OJ with $\mathrm{Sm}$ and $1.34 \pm 0.04$ of KC with $\mathrm{Sm}$. The $\mathrm{pH}$ of the mixtures remained between 3.5 and 4.25 , within the levels found by other authors (Barros et al., 2011). However, the TSS with WEP was higher in all cases than in control matrices, and they coincide with the sweetness value given by judges. Therefore, while the TSS of the control OJ did not exceed $10.67{ }^{\circ}$ Brix, when the juice was fortified with WEP this parameter was higher, ranging between $11.11 \pm 0.15$ in freshly made OJ with $S m$ and $11.83 \pm 0.11$ in OJ with $Q b$ after $24 \mathrm{~h}$ of storage at $20^{\circ} \mathrm{C}$. The TSS of $\mathrm{KC}$ was also higher in matrices enriched with WEP than in control KC. While TSS of control KC did not exceed $13.93{ }^{\circ}$ Brix, the TSS of the fortified mixtures of kiwifruit ranged between $14.30 \pm 0.15^{\circ}$ Brix of freshly made KC with $\mathrm{Sm}$ and $14.94 \pm 0.24$ of $\mathrm{KC}$ with $\mathrm{Qb}$ after $24 \mathrm{~h}$ of storage at $20^{\circ} \mathrm{C}$. In general, the beverages minimally processed with higher TSS were the matrices fortified with $Q b$, the same that were considered sweetest by the judges (Table 2). This increase of TSS is an interesting characteristic because it can increase the sweetness of the beverages without adding sugars and, therefore, avoiding the increase of empty calories (Guenther, Reedy, \& Krebs-Smith, 2008), a more and more demanded issue by consumers.

\subsection{Changes in colour quality}

The enrichment of OJ and KC with WEP with high antioxidant capacity can change colour, although it can also contribute to its

\section{Table 2}

Acidity, $\mathrm{pH}$ and soluble solids of orange juice (OJ) and kiwifruit cremogenate (KC) after addition of wild edible plants (WEP).

\begin{tabular}{lclll}
\hline Matrix with WEP & Time $(\mathrm{h})^{\mathrm{b}}$ & $\mathrm{TA}\left(\mathrm{g} 100 \mathrm{~g}^{-1}\right)$ & $\mathrm{pH}$ & $\mathrm{TSS}($ Brix $)$ \\
\hline OJ Control & 0 & $1.61 \pm 0.02$ & $3.61 \pm 0.20$ & $10.63 \pm 0.11$ \\
OJ with $R c$ & & $1.54 \pm 0.03$ & $3.73 \pm 0.03$ & $11.10 \pm 0.12$ \\
OJ with $Q b$ & & $1.58 \pm 0.05$ & $3.74 \pm 0.07$ & $11.43 \pm 0.07$ \\
OJ with $S m$ & & $1.66 \pm 0.04$ & $3.45 \pm 0.30$ & $11.01 \pm 0.15$ \\
OJ Control & 24 & $1.65 \pm 0.01$ & $3.64 \pm 0.14$ & $10.67 \pm 0.22$ \\
OJ with $R c$ & & $1.57 \pm 0.03$ & $3.43 \pm 0.02$ & $11.30 \pm 0.10$ \\
OJ with $Q b$ & & $1.60 \pm 0.04$ & $3.39 \pm 0.02$ & $10.50 \pm 0.06$ \\
OJ with $S m$ & $1.63 \pm 0.08$ & $3.57 \pm 0.06$ & $10.83 \pm 0.09$ \\
\hline LSD $(p<0.05)^{\mathrm{c}}$ & & 0.06 & 0.20 & 0.18 \\
\hline KC Control & 0 & $1.40 \pm 0.01$ & $3.67 \pm 0.42$ & $13.53 \pm 0.12$ \\
KC with $R c$ & & $1.41 \pm 0.06$ & $4.07 \pm 0.13$ & $14.78 \pm 0.38$ \\
KC with $Q b$ & & $1.34 \pm 0.05$ & $3.61 \pm 0.26$ & $14.38 \pm 0.23$ \\
KC with $S m$ & & $1.34 \pm 0.04$ & $4.25 \pm 0.26$ & $14.33 \pm 0.07$ \\
KC Control & 24 & $1.39 \pm 0.02$ & $3.6 \pm 0.23$ & $13.93 \pm 0.18$ \\
KC with $R c$ & & $1.44 \pm 0.03$ & $3.74 \pm 0.29$ & $14.87 \pm 0.30$ \\
KC with $Q b$ & & $1.37 \pm 0.04$ & $3.70 \pm 0.29$ & $14.43 \pm 0.24$ \\
KC with $S m$ & & $1.35 \pm 0.03$ & $3.64 \pm 0.25$ & $14.30 \pm 0.15$ \\
\hline$L S D(p<0.05)^{c}$ & & 0.05 & 0.39 & 2.23 \\
\hline
\end{tabular}

$\overline{\text { Values represent the means } \pm \text { SD of three replications for each of three sub-samples }}$ per sample.

a Matrix: Orange juice (OJ), kiwifruit cremogenate (KC) fortified with WEP (Rosa canina -Rc-, Quercus ballota-Qb- or Sanguisorba minor-Sm-).

b Time ( $0 \mathrm{~h}$ and after $24 \mathrm{~h}$ a $\left.20{ }^{\circ} \mathrm{C}\right)$.

$L S D(p<0.05)$, least significant difference, was used for significant difference comparison among means of various samples. stability against oxidation. In OJ, the addition of $R c$ significantly increased $(p<0.05)$ the orange colour of the juice by increasing the redness (CIE $a^{*}$ value) and the yellowness (CIE $b^{*}$ value) (Table 3$)$. On the contrary, the addition of $\mathrm{Qb}$ did not change the CIA $a^{*}$, although it increased the lightness (CIEL $L^{*}$ value) and decreased the intensity of the orange colour. The addition of $\mathrm{Sm}$ significantly changed $(p<0.05)$ colour (CIEa* more negative than that of control) due to the chlorophyll of leaves and young shoots of this plant that gives the juice a colour less valued by consumers (Table 2). It is worth mentioning that the addition of $R c$ to $\mathrm{KC}$ gave a brownish colour to the mixture $\left(\mathrm{CIEa}^{*}-4.53 \pm 0.04\right.$ and Hue angle $\left.105.36 \pm 0.09\right)$, coincident with a low value given by consumers (61.87\% regarding control). However, the resulting colour of the mixture of $Q b$ or $S m$ with $\mathrm{KC}$, with lower $\mathrm{CIE} a^{*}$ and $\mathrm{CIE} b^{*}$ values, was better valued by consumers than that of control (Table 2). The mixtures of different matrices change the profile of the pigments (Lemmens, Van Buggenhout, Van Loey, \& Hendrickx, 2010). So WEP with high contents of chlorophyll and brown pigments are more suitable for $\mathrm{KC}$, while those rich in carotenoids (orange and red pigments) are more suitable for $\mathrm{OJ}$ in order to obtain a product well valued by consumers.

After $24 \mathrm{~h}$ at $20^{\circ} \mathrm{C}$, the orange colour of OJ was significantly $(p<0.05)$ less intense, as it can be shown by the decrease of CIEa* and $\mathrm{CIEb} b^{*}$ (Table 3 ) probably caused by the degradation of $\beta$-cryptoxanthin, antheraxanthin and violaxanthin, the main responsible carotenoids for the colour of the orange juice (Carmona, Zacarias, \& Rodrigo, 2012). Although the addition of $R c$ to OJ did not avoid the degradation of carotenoids of the juice, the OJ with $R c$ preserved an intense red colour due to the increase in the mixture of $\beta$-carotene found in R. canina (97.77 $\mathrm{mg} 100 \mathrm{~g}^{-1} \mathrm{DW}$ ) (Barros et al., 2011). It is already known that the stability of red colour is a big problem in food processing (Rein \& Heinomen, 2004) and, therefore, the addition of $R c$ as colouring agent to certain matrices could compensate for the loss of pigments during the processing and could enrich the juices with other bioactive compounds found in R. canina (Barros et al., 2011; Egea et al., 2010). On the other side, the addition of $Q b$ to the OJ implied a quicker loss of red colour of the OJ regarding control, while the OJ with $\mathrm{Sm}$ practically reached the same colour than the control one, it won redness (less negative $\mathrm{CIE} a^{*}$ value). Therefore, it seems that $Q b$ potentiates the degradation of the characteristic carotenoids of the orange juice and the OJ potentiates the degradation of the chlorophyll of $\mathrm{Sm}$.

The colour of $\mathrm{KC}$ changed after $24 \mathrm{~h}$ at $20^{\circ} \mathrm{C}$, with a significant decrease of the Hue angle (more than $70^{\circ}$ of difference between initial and final values), a fact showing a high percentage of darkening (González-Molina et al., 2008). The addition of WEP to KC affects in different ways according to the species. While the addition of $S m$ does not reduce darkening (Hue angle value similar to the control), the addition of $R c$ and $Q b$ significantly reduce darkening of KC and, particularly, the addition of $R c$ reduce darkening to half. Therefore, $Q b$ and $R c$ could be used as an alternative to synthetic compounds for minimizing the darkening in other products that are minimally processed like vegetables cremogenate. However, the use of these species as anti-darkening agents would need a deeper study because the colour stabilization depends upon the structure and concentration of the pigment, $\mathrm{pH}$, temperature, presence of co-pigments, ascorbic acid, metallic ions, etc (Wicklund et al., 2005).

\subsection{Changes in vitamin $C$ and polyphenols contents}

The addition of WEP to OJ did not significantly change $(p<0.05)$ the levels of vitamin $C$ (ascorbic acid plus dehydroascorbic acid) regarding the non fortified $\mathrm{OJ}$ and $\mathrm{KC}$ matrices (Fig. 1a, c). The initial levels of vitamin C in OJ were $124.75 \pm 3.35 \mathrm{mg} 100 \mathrm{~g}^{-1} \mathrm{FW}$, similar 
Table 3

Colour of orange juice (OJ) and kiwifruit cremogenate (KC) after enrichment with wild edible plants (WEP).

\begin{tabular}{|c|c|c|c|c|c|c|}
\hline Matrix with WEP & Time $(h)^{b}$ & $\mathrm{CIEL} L^{*}$ & $\mathrm{CIE} a^{*}$ & $\mathrm{CIE} b^{*}$ & Croma & Hue \\
\hline OJ Control & 0 & $38.08 \pm 0.28$ & $-5.58 \pm 0.04$ & $18.47 \pm 0.45$ & $19.30 \pm 0.44$ & $106.83 \pm 0.27$ \\
\hline OJ with $R c$ & & $39.82 \pm 0.19$ & $-2.78 \pm 0.01$ & $20.26 \pm 0.09$ & $20.45 \pm 0.08$ & $97.81 \pm 0.02$ \\
\hline OJ with $Q b$ & & $43.41 \pm 0.27$ & $-5.38 \pm 0.03$ & $22.37 \pm 0.31$ & $23.01 \pm 0.31$ & $103.53 \pm 0.15$ \\
\hline OJ with $\mathrm{Sm}$ & & $38.22 \pm 0.18$ & $-7.55 \pm 0.14$ & $17.24 \pm 0.44$ & $18.83 \pm 0.45$ & $113.66 \pm 0.18$ \\
\hline OJ Control & 24 & $38.82 \pm 0.12$ & $-5.92 \pm 0.12$ & $17.92 \pm 0.37$ & $18.87 \pm 0.39$ & $108.29 \pm 0.21$ \\
\hline OJ with $R c$ & & $39.58 \pm 0.33$ & $-2.96 \pm 0.08$ & $19.04 \pm 0.18$ & $19.27 \pm 0.19$ & $98.82 \pm 0.15$ \\
\hline OJ with $Q b$ & & $42.85 \pm 0.12$ & $-6.15 \pm 0.19$ & $20.80 \pm 0.53$ & $21.70 \pm 0.53$ & $106.49 \pm 0.47$ \\
\hline OJ with $\mathrm{Sm}$ & & $38.74 \pm 0.78$ & $-5.41 \pm 0.24$ & $17.36 \pm 0.89$ & $18.18 \pm 0.92$ & $107.32 \pm 0.18$ \\
\hline$L S D(p<0.05)^{c}$ & & 0.49 & 0.18 & 0.65 & 0.66 & 0.33 \\
\hline KC Control & 0 & $43.75 \pm 0.70$ & $-7.41 \pm 0.08$ & $16.12 \pm 0.22$ & $17.74 \pm 0.22$ & $114.69 \pm 0.32$ \\
\hline KC with $R c$ & & $42.70 \pm 0.03$ & $-4.53 \pm 0.04$ & $16.48 \pm 0.21$ & $17.09 \pm 0.22$ & $105.36 \pm 0.09$ \\
\hline$K C$ with $Q b$ & & $47.08 \pm 0.12$ & $-7.76 \pm 0.05$ & $18.06 \pm 0.20$ & $19.60 \pm 0.21$ & $113.32 \pm 0.11$ \\
\hline KC with Sm & & $42.41 \pm 0.50$ & $-9.14 \pm 0.17$ & $18.75 \pm 0.12$ & $20.86 \pm 0.15$ & $115.98 \pm 0.38$ \\
\hline KC Control & 24 & $38.96 \pm 0.27$ & $-2.77 \pm 1.23$ & $11.39 \pm 0.38$ & $19.25 \pm 1.00$ & $44.64 \pm 1.82$ \\
\hline KC with $R c$ & & $39.00 \pm 0.36$ & $-2.27 \pm 0.27$ & $13.24 \pm 0.26$ & $32.34 \pm 7.32$ & $71.84 \pm 12.81$ \\
\hline$K C$ with $Q b$ & & $43.42 \pm 0.41$ & $-4.42 \pm 0.10$ & $13.26 \pm 0.62$ & $34.29 \pm 7.61$ & $62.81 \pm 16.06$ \\
\hline KC with Sm & & $39.00 \pm 0.36$ & $-2.27 \pm 0.27$ & $13.24 \pm 0.26$ & $18.26 \pm 1.31$ & $48.49 \pm 11.03$ \\
\hline $\operatorname{LSD}(p<0.05)^{c}$ & & 0.55 & 0.65 & 0.44 & 5.28 & 11.55 \\
\hline
\end{tabular}

Values represent the means \pm SD of three replications for each of three sub-samples per sample.

a Matrix: Orange juice (OJ), Kiwifruit cremogenate (KC) fortified with WEP (Rosa canina -Rc-, Quercus ballota -Qb- o Sanguisorba minor -Sm-).

b Time ( $0 \mathrm{~h}$ and after $24 \mathrm{~h}$ at $\left.20^{\circ} \mathrm{C}\right)$.

${ }^{c} \operatorname{LSD}(p<0.05)$, least significant difference, was used for significant difference comparison among means of various samples.

to those found by other authors (Tarrago-Trani, Philips, \& Cotty, 2012). Although the addition of WEP increases vitamin $C$ between 10 and 15\%, it was not a significant increase. Vitamin C in KC did not change regarding control when WEP were added; the levels ranged around $170 \mathrm{mg} 100 \mathrm{~g}^{-1} \mathrm{FW}$, similar to those found by other authors (Celik, Sezai, \& Turgut, 2007). The stability of vitamin C in matrices (OJ and $\mathrm{KC}$ ) at $20{ }^{\circ} \mathrm{C}$ during $24 \mathrm{~h}$ did not significantly change $(p<0.05)$. So, in general, the addition of WEP to OJ and KC
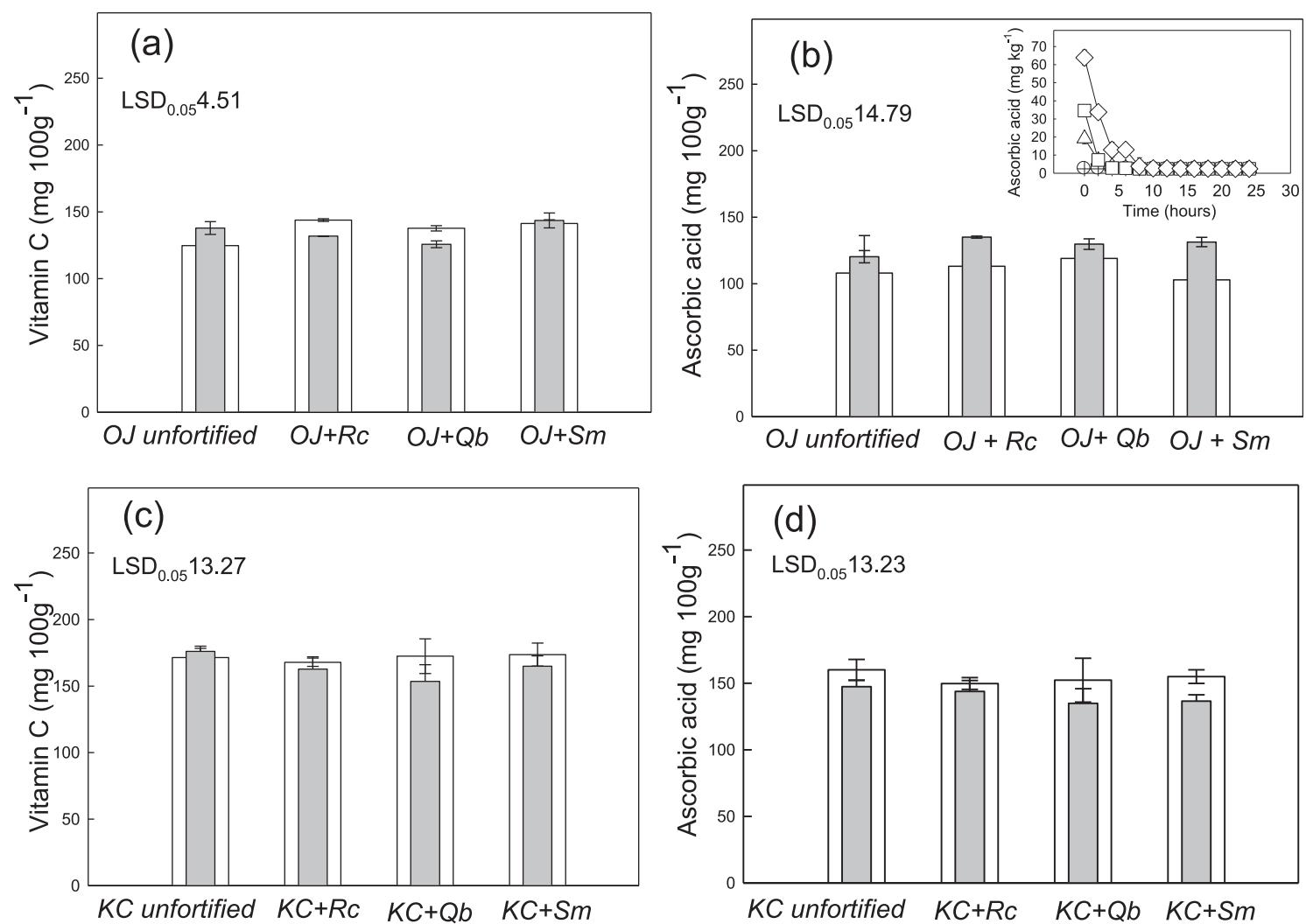



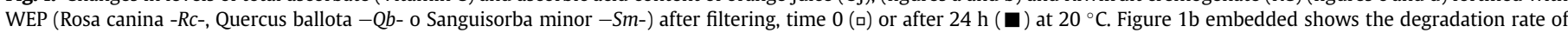

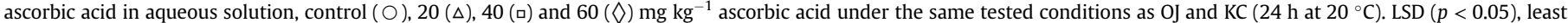

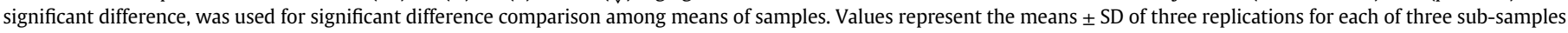
per sample. 
did not significantly change the content of vitamin $\mathrm{C}$ or increase its stability under tested conditions. For nutritional purposes, vitamin $C$ is the sum of AA and dehydroascorbic acid (DHAA); DHAA absorbed from the intestinal tract undergoes intracellular reduction (via NADPH- and glutathione-dependent reductases), rendering it biologically active (González-Molina et al., 2008). It must be mentioned that when the matrix is OJ, the proportion of AA (Fig. 1 b) after $24 \mathrm{~h}$ at $20^{\circ} \mathrm{C}$ significantly increased $(p<0.05)$. AA is susceptible to oxidation in aqueous solution; therefore, temperature, light, $\mathrm{pH}$, the presence of oxygen, metal catalysts (iron, copper) and enzymes such as ascorbate oxidase affect the rate of AA degradation, at a variable rate in different foods (Johnston, Steinberg, \& Rucker, 2007). It was verified (Fig. 1b embedded) that, in aqueous solution and under tested conditions (24 h at $20^{\circ} \mathrm{C}$ ), the speed of degradation of the ascorbic acid is very high during the first hours, regardless the concentration of AA of the initial solution.

The ascorbic acid in OJ or KC (Fig. 1 b, d) did not significantly degrade $(p<0.05)$ after $24 \mathrm{~h}$ at $20^{\circ} \mathrm{C}$. The TA and the $\mathrm{pH}$ of the matrices contribute to the AA stability because at low pH AA is fully protonated and relatively stable, with maximal stability at 4-6; at $\mathrm{pH}>6, \mathrm{AA}$ is reversibly oxidized to DHAA, and DHAA may be further irreversibly oxidized to 2,3-diketo-l-gluconic acid, followed by degradation to other by-products (Johnston et al., 2007). On the other side, $\mathrm{OJ}$ is a rich source of flavanons, including hesperidin and narirutin, which are also main phenolic compounds of OJ (Proteggente et al., 2002). Moreover, kiwifruit is rich in polyphenols compared with other fruits (Park et al., 2006). The addition of $R c$ and $\mathrm{Sm}$ increased the content of phenolic compounds in both matrices (OJ and $\mathrm{KC}$ ) in more than $30 \%$ due to the fact that both $R c$ and $\mathrm{Sm}$ have a high concentration of these compounds (Barros et al., 2011; Egea et al., 2010). However, the addition of $Q b$ did not significantly change the levels of polyphenols (Fig. 2a, b). The high concentration of tannins found in $Q b$ could cause the precipitation of tannin acids or tannin-protein complexes responsible for the astringent flavour of the tannins, a flavour that is disgusting to the consumer (Tejerina et al., 2011). The phenolic compounds remained stable during $24 \mathrm{~h}$ at room temperature in all matrices.

\subsection{Changes in Trolox equivalent antioxidant capacity (TEAC) and relationship with phytonutrient levels}

One of the main quality attributes of juices is their antioxidant capacity and, during the last years, this has been a very solid argument for the professionals of the food industry. Fig. 2 (c and d) shows the antioxidant capacity (TEAC) of OJ and KC, respectively, after their enrichment with different WEP. The initial antioxidant capacity of KC was higher than that of OJ $(359.04 \pm 46$ and $298.07 \pm 10.2$ equi Trolox $\mu \mathrm{mol} \mathrm{L}^{-1}$, respectively). There is a controversial debate in the literature about the influence of vitamin $\mathrm{C}$ on the antioxidant capacity of fruits or vegetables (Guo et al., 2003; Tavarini et al., 2008). It is also known that fruits with high antioxidant capacity generally contain more antioxidants and most of these antioxidants has been showed to be phenolic compounds and, in particular, flavonoids (Guo et al., 2003). According to some authors (Proteggente et al., 2002; Tavarini et al., 2008), the antioxidant capacity of OJ mainly results from its ascorbic acid content, while flavanon glycosides have a limited contribution to its antioxidant capacity. In our work, the addition of $Q b$ did not significantly increase the antioxidant capacity of the matrices (OJ and $\mathrm{KC}$ ), while the addition of $R c$ increased in 67 and 39\% the antioxidant capacity of $\mathrm{OJ}$ and $\mathrm{KC}$, respectively. The antioxidant capacity of OJ
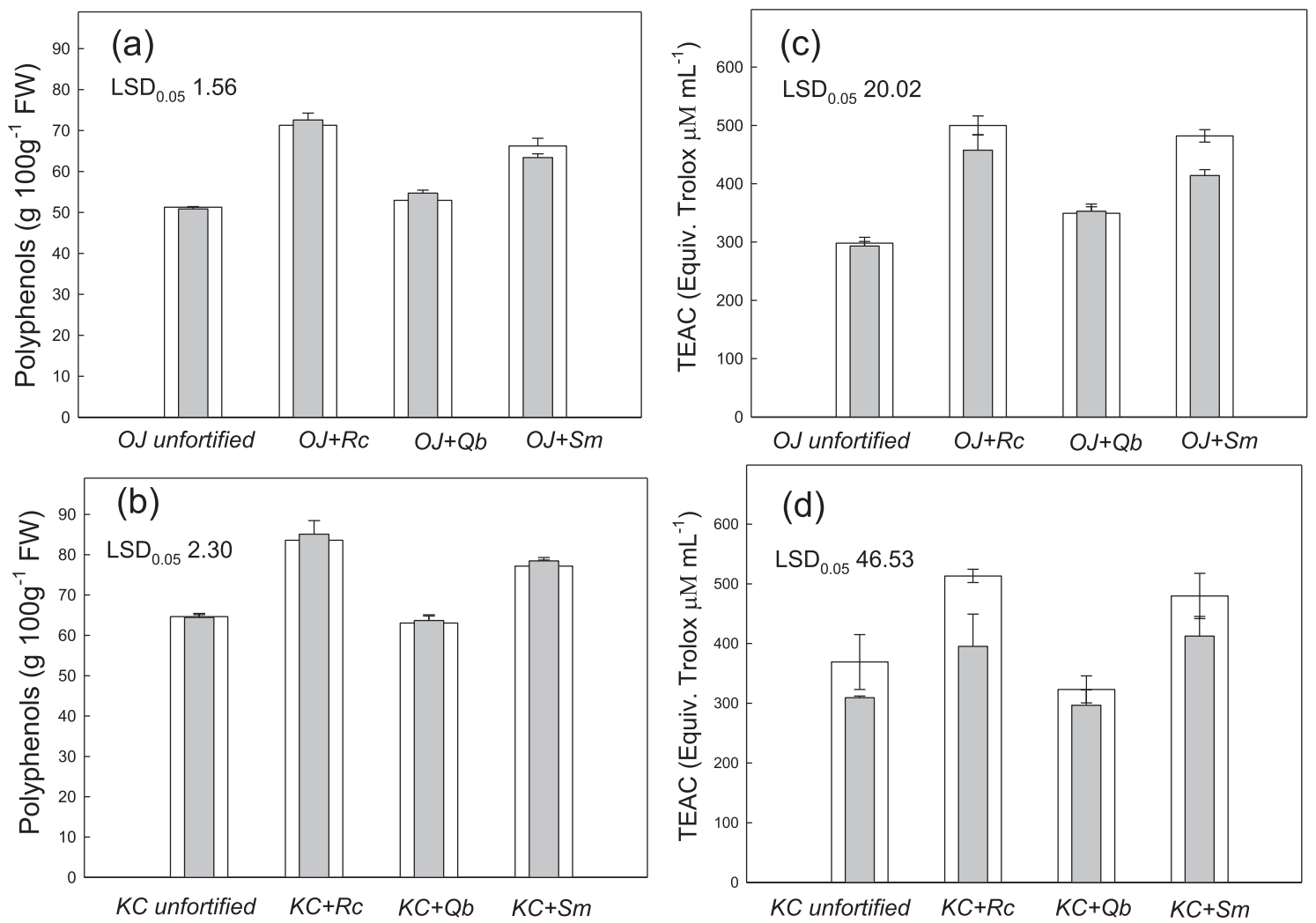



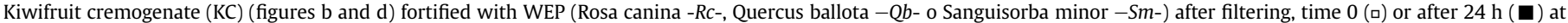

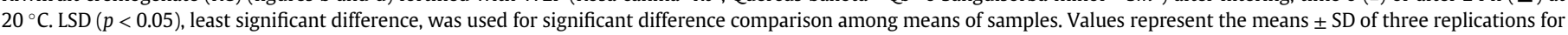
each of three sub-samples per sample. 
Table 4

Analysis of correlations between total antioxidant activity (TEAC), total polyphenols content and vitamin $\mathrm{C}$ in the prepared mixtures.

\begin{tabular}{lllll}
\hline \multicolumn{2}{l}{ Antioxidant capacity (TEAC) } & & \\
\hline & $\mathrm{OJ}^{0 \mathrm{~h}}$ & $\mathrm{KC}^{0 \mathrm{~h}}$ & $\mathrm{OJ}^{24 \mathrm{~h}}$ & $\mathrm{KC}^{24 \mathrm{~h}}$ \\
\hline Phenolics & $0.9620^{\mathrm{MS}}$ & $0.9669^{\mathrm{MS}}$ & $0.9500^{\mathrm{MS}}$ & $0.9280^{\mathrm{MS}}$ \\
Vitamin C & 0.3095 & 0.5220 & 0.1988 & 0.2091 \\
\hline
\end{tabular}

MS Marginally significant $(P>0.05)$.

and KC with Sm also increased in 60 and 30\%, respectively. Coincident to our results, some studies have shown that the activity in the TEAC assay increases with the number of hydroxyl groups of the antioxidant compound, so the antioxidant activity is higher for phenolic substances than for ascorbic acid, in which only one hydroxyl group reacts under experimental conditions (Hodisan, Socaciu, Ropan, \& Neamtu, 1997). This relation between total phenols and the antioxidant activity has been found in many fruits and all their derived products (Egea et al., 2010), a fact suggesting that the phenolic compounds could be responsible for the antioxidant properties of the new beverages fortified with $R c$ and $S m$ because there is a good correlation (Table 4) between the content in polyphenols of the new beverages and the antioxidant capacity and, however, there is a very low correlation between the content in vitamin $C$ and the antioxidant capacity of the mixtures.

However, when the new beverages were stored during $24 \mathrm{~h}$ at $20^{\circ} \mathrm{C}$ the antioxidant capacity of the beverages fortified with $R c$ and $\mathrm{Sm}$ significantly decreased, between 10 and 15\% in OJ and between 15 and $25 \%$ in KC. This decrease could be due to reactions of polymerization, condensation or complexation that can cause the formation of new compounds with a lower antioxidant capacity (González-Molina et al., 2008). Moreover, the presence of other antioxidant compounds from $R c$ and $S m$, such as proteins, which we have not analysed, could react in the mixtures decreasing the in vitro antioxidant values (González-Molina et al., 2008). The antioxidant properties of food matrices are due to the presence in complex mixtures of compounds of varying polarity, such as vitamin C, vitamin E, carotenoids and polyphenols. Due to the potential synergistic action of all these bioactive compounds present in food (Egea et al., 2010) the total antioxidant capacity of some foods has increasingly become a sales argument for professionals in the food industry.

\section{Conclusion}

The beverages that result from the addition of wild edible plants with high antioxidant capacity to orange juice or kiwifruit cremogenate are well accepted by consumers, even improving the acceptance of the fresh product, and they improve the content in phenolic compounds and the antioxidant capacity without changing the physico-chemical properties of the matrices. This better acceptance of enriched beverages minimally processed by the consumer was due, to a great extent, to the intensification of the natural colour (in the case of addition of $R c$ to OJ and $S m$ to KC) and to the extra sweetness provided mainly by $Q b$ and, to a lesser extent, by $R c$ because the addition of wild edible plants to the matrices increased their content in soluble solids. Beverages minimally processed with a high interest for consumers and with high antioxidant properties have been obtained.

\section{References}

Barros, L., Carvalho, A. M., \& Ferreira, I. (2011). Exotic fruits as a source of importan phytochemicals: improving the traditional use of Rosa canina fruits in Portugal. Food Research International, 44(7), 2233-2236.

Benlloch-Tinoco, M., Igual, M., Rodrigo, D., \& Martínez-Navarrete, N. (2013). Comparison of microwaves and conventional thermal treatment on enzymes activity and antioxidant capacity of kiwifruit puree. Innovative Food Science and Emerging Technologies, 19, 166-172.

Boğa, M., Hacıbekiroğlu, I., \& Kolak, U. (2011). Antioxidant and anticholinesterase activities of eleven edible plants. Pharmaceutical Biology, 49(3), 290-295.

Carmona, L., Zacarias, L., \& Rodrigo, M. J. (2012). Stimulation of coloration and carotenoid biosynthesis during postharvest storage of 'Navelina' orange fruit at $12{ }^{\circ} \mathrm{C}$. Postharvest Biology and Technology, 74, 108-117.

Cassano, A., \& Drioli, E. (2007). Concentration of clarified kiwifruit juice by osmotic distillation. Journal of Food Engineering, 79, 1397-1404.

Celik, A., Sezai, E., \& Turgut, N. (2007). Some physical, pomological and nutritional properties of kiwifruit cv. Hayward. International Journal of Food Sciences and Nutrition, 58, 411-418.

Egea, I., Sánchez-Bel, P., Romojaro, F., \& Pretel, M. T. (2010). Six edible wild fruits as potential antioxidant additives or nutritional supplements. Plant Foods For Human Nutrition, 65, 121-129.

Gatto, M. A., Ippolito, A., Linsalata, V., Cascarano, N. A., Nigro, F., Vanadia, S., et al. (2011). Activity of extracts from wild edible herbs against postharvest fungal diseases of fruit and vegetables. Postharvest Biology and Technology, 61(1), 72-82.

González-Molina, E., Moreno, D. A., \& García-Viguera, C. (2008). Aronia-enriched lemon juice: a new highly antioxidant beverage. Journal of Agricultural and Food Chemistry, 56, 11327-11333.

Guenther, P. M., Reedy, J., \& Krebs-Smith, S. M. (2008). Development of the healthy eating index. Journal of the American Dietetic Association, 108(11), 1896-1901.

Guo, C. J., Yang, J. J., Wei, J. Y., Li, Y. F., Xu, J., \& Jiang, Y. G. (2003). Antioxidant activities of peel, pulp and seed fractions of common fruits as determined by FRAP assay. Nutrition Research, 23, 1719-1726.

Hodisan, T., Socaciu, C., Ropan, I., \& Neamtu, G. (1997). Carotenoid composition of Rosa canina fruits determined by thin-layer chromatography and highperformance liquid chromatography. Journal of Pharmaceutical and Biomedical Analysis, 16, 521-528.

Janzantti, N. S., Machado, T. V., \& Monteiro, M. (2011). Sensory acceptance of juice from FCOJ processing steps. Journal of Sensory Studies, 26(5), 322-330.

Johnston, C. S., Steinberg, F. M., \& Rucker, R. B. (2007). Ascorbic acid. In J. Zempleni, R. B. Rucker, D. B. McCormick, \& J. W. Suttie (Eds.), Handbook of vitamins (4th ed.) (pp. 489-520). Boca Raton, FL, USA: CRC Press.

Lattanzio, F., Greco, E., Carretta, D., Cervellati, R., Govonic, P., \& Speronia, E. (2011). In vivo anti-inflammatory effect of Rosa canina L. extract. Journal of Ethnopharmacology, 137, 880-885.

Lavecchia, T., Rea, G., Antonacci, A., \& Giardi, M. T. (2013). Healthy and adverse effects of plant-derived functional metabolites: the need of revealing their content and bioactivity in a complex food matrix. Critical Reviews in Food Science and Nutrition, 53, 198-213.

Lee, M. H., Jeong, J. H., \& Oh, M. J. (1992). Antioxidative activity of gallic acid in acorn extract. Journal of the Korean Society of Food and Nutrition, 21(6), 693-700.

Lemmens, L., Van Buggenhout, S., Van Loey, A. M., \& Hendrickx, M. E. (2010). Particle size reduction leading to cell wall rupture is more important for the $\beta$-carotene bioaccessibility of raw compared to thermally processed carrots. Journal of Agricultural and Food Chemistry, 58, 12769-12776.

Mandade, R. J., Choudhury, A., Harsulkar, A., \& Wakade, R. (2011). Role of the Rosa canina L. leaf extract as an antidiarrheal drug in rodents. Indian Journal of Pharmacology, 43, 316-319.

Pala, Ç. U., \& Toklucu, A. K. (2013). Microbial, physicochemical and sensory properties of UV-C processed orange juice and its microbial stability during refrigerated storage. LWT - Food Science and Technology, 50(2), 426-431.

Pardo de Santayana, M., Tardío, J., \& Morales, R. (2005). The gathering and consumption of wild edible plants in the Campoo (Cantabria, Spain). International Journal of Food Sciences and Nutrition, 56(7), 529-542.

Park, Y. S., Junj, S. T., Kang, S. G., Drzewiecki, J., Namiesnik, J., Haruenkit, R., et al. (2006). In vitro studies of polyphenols, antioxidants and other dietary indices in kiwifruit (Actinidia deliciosa). International Journal of Food Sciences and Nutrition, 57, 107-122.

Proteggente, A. R., Pannala, A. S., Paganga, G., Van Buren, L., Wagner, E., \& Wiseman, S. (2002). The antioxidant activity of regularly consumed fruit and vegetables reflects their phenolic and vitamin C composition. Free Radical Research, 36(2), 217-233.

Rein, M. J., \& Heinomen, M. (2004). Stability and enhancement of berry juice color. Journal of Agricultural and Food Chemistry, 52(10), 3106-3114.

Romojaro, A., Botella, M. A., Obón, C., \& Pretel, M. T. (2013). Nutritional and antioxidant properties of wild edible plants and their use as potential ingredients in the modern diet. International Journal of Food Sciences and Nutrition, 64(8), 944-952.

Sanchez-Bel, P., Egea, I., Romojaro, F., \& Martinez-Madrid, M. C. (2008). Sensorial and chemical quality of electron beam irradiated almonds (Prunus amygdalus). LWT-Food Science and Technology, 41, 442-449.

Serrano, M., Diaz-Mula, H. M., Zapata, P. J., Castillo, S., Guillen, F., MartinezRomero, D., et al. (2009). Maturity stage at harvest determines the fruit quality and antioxidant potential after storage of sweet cherry cultivars. Journal of Agricultural and Food Chemistry, 57, 3240-3246.

Tarrago-Trani, M. T., Philips, K. M., \& Cotty, M. (2012). Matrix-specific method validation for quantitative analysis of vitamin $C$ in diverse foods. Journal of Food Composition and Analysis, 26, 12-25.

Tavarini, S., Degl'Innocentia, E., Remorinib, D., Massaib, R., \& Guidi, L. (2008). Antioxidant capacity, ascorbic acid, total phenols and carotenoids changes during harvest and after storage of Hayward kiwifruit. Food Chemistry, 107, $282-288$. 
Tejerina, D., García-Torres, S., Cabeza de Vaca, M., Vázquez, F. M., \& Cava, R. (2011). Acorns (Quercus rotundifolia Lam.) and grass as natural sources of antioxidants and fatty acids in the "montanera" feeding of Iberian pig: intra- and interannual variations. Food Chemistry, 124, 997-1004.

Tepper, B. J. (1993). Effects of a slight color variation on consumer acceptance of orange juice. Journal of Sensory Studies, 8, 145-154.
Wicklund, T., Rosenfeld, H. J., Martinsen, B. K., Sundfør, M. W., Lea, P., Bruun, T., et al. (2005). Antioxidant capacity and colour of strawberry jam as influenced by cultivar and storage conditions. LWT-Food Science and Technology, 38, 387-391. Yilmaz, S. O., \& Ercisli, S. (2011). Antibacterial and antioxidant activity of fruits of some rose species from Turkey. Romanian Biotechnological Letters, 16 6407-6411.

Please cite this article in press as: Sanchez-Bel, P., et al., Wild edible plants as potential antioxidant or nutritional supplements for beverages minimally processed, LWT - Food Science and Technology (2014), http://dx.doi.org/10.1016/j.lwt.2014.06.017 